\title{
Optimierte Versorgung schwerer Wunden
}

— Eine moderne Wundversorgung sollte die Wundheilung und die Lebensqualität verbessern und an die Bedürfnisse der Patienten angepasst sein. Die neue Wundauflage Urgotül ${ }^{\circledR}$ Soft, eine Weiterentwicklung von Urgotül ${ }^{\circledR}$, komme dem Ziel einer patientenorientierten Wundversorgung sehr nahe, so Dr. Katharina Herberger, Hamburg. Sie besteht aus einem Polyestergitter mit einer Lipokolloidauflage und ist getränkt mit Hydrokolloidpartikeln und Vaseline. Durch die Struktur eines imprägnierten und offenporigen Gitters ist sie anpassungsfähig, elastisch und sehr flexibel. Durch elastische Fäden in der Gitterstruktur kann sich die Wundauflage der Wunde anpassen selbst bei ausgeprägten Rundungen oder zerklüfteten Wundflächen. Aufgrund der hohen Flexibilität lasse sich die Wundauflage sogar über Gelenke hinweg aufbringen, so dass die Beweglichkeit erhalten bleibe, erläuterte Herberger. Formbeständige Maschen ermöglichen das Abfließen des Exsudats.
Indikationen für Urgotül ${ }^{\circledR}$ Soft sind akute Wunden wie Verbrennungen, Hautabschürfungen und traumatische Verletzungen, aber auch chronische Läsionen wie Ulzera oder Dekubiti sowie Hautschädigungen bei Epidermolysis bullosa. Wegen der guten Modellierbarkeit könne das Produkt aber auch zum Tamponieren zerklüfteter Wunden verwendet werden, so Herberger.

aam

Launch-Pressegespräch „UrgoCell ${ }^{\circledR}$ START Contact und Urgotül ${ }^{\circledR}$ Soft: Neue Wege in der modernen Wundversorgung", Berlin, 7.10.2009 (Veranstalter: Urgo).

\section{„Optische Biopsie“" klärt Befunde ohne Skalpell}

- Mit der konfokalen Laserscan-Mikroskopie lassen sich malignomverdächtige dermatoskopische Befunde nicht-invasiv abklären. Mobile In-vivo-Geräte ermöglichen einen komfortablen Einsatz - auch ambulant.

VivaScope 1500 und VivaSope 3000 heißen die beiden Geräte, die das Unternehmen MAVIG beim 18. EADV-Kongress in Berlin präsentiert hat. Während bei dem $1500 e r-$ Modell die Lichtquelle an einem SchwenkArm untergebracht ist, ist das zoooer-Modell ein ultramobiles Handgerät, mit dem auch schwer zugängliche Hautpartien problemlos erreichbar sind. „Die Geräte arbeiten mit Licht der Wellenlänge $830 \mathrm{~nm}$, was eine vollkommen schmerzfreie Untersuchung verdächtiger Hautbefunde ermöglicht", erläuterte Martina Ulrich von der Klinik für Dermatologie der Charité Berlin. Das Gerät liefert auf einem Monitor histologieähnliche Schwarz-Weiß-Bilder, allerdings als Horizontalschnitte. Die Eindringtiefe liegt bei $250 \mu \mathrm{m}$, reicht also etwa bis zum Stratum reticulare der Dermis.

Das Verfahren eigne sich sowohl für weiße wie für schwarze Hautkrebsläsionen, so Ulrich. Der Hauptvorteil liege in der Geschwindigkeit der Diagnose. Den Patienten bleiben Wartezeiten von bis zu zwei Wochen erspart. Um die Ärzte im Umgang mit konfokalen Laserbildern zu schulen, bietet der Hersteller zwei Schulungsprogramme für weißen Hautkrebs (in Berlin) und für schwarzen Hautkrebs (in Modena) an. Zusätzlich gibt es weitere Materialien und auch eine Online-Schulung.

In Deutschland werden die VivaScope-Geräte bereits von vier niedergelassenen Hautärzten und einer Reihe von Unikliniken eingesetzt. Die Kosten liegen bei 80.000 Euro pro Gerät. Die konfokale Laserscanmikroskopie ist derzeit keine Kassenleistung. gvg

Symposium „Konfokale Laserscanmikroskopie mit VivaScope Systems - Innovation für Analyse, Diagnose und therapeutisches Monitoring“, 10.10.2009 (Veranstalter: MAVIG).

\section{Infliximab bewährt sich bei Plaque-Psoriasis}

_ Patienten mit mittelschwerer bis schwerer Plaque-Psoriasis profitieren von einer Behandlung mit dem TNS-Antikörper Infliximab (Remicade ${ }^{\circledR}$ ). Wie die große randomisierte Vergleichsstudie RESTORE1 zeigt, ist die Wirksamkeit des TNF-Antikörpers einer Therapie mit Methotrexat (MTX) signifikant überlegen. In der Multizenterstudie, die im Rahmen des EADV-Kongresses 2009 vorgestellt wurde, wurden 868 Patienten, die seit mindestens sechs Monaten an einer Plaque-Psoriasis litten (PASI $\geq$ $12, \geq 10 \%$ der Körperfläche) entweder mit $5 \mathrm{mg} / \mathrm{kg}$ Infliximab in den Wochen o, 2, 6, 14 und 22 behandelt oder sie erhielten MTX in einer Dosierung von initial $15 \mathrm{mg} /$ Woche, die bei Bedarf $\mathrm{ab}$ Woche 6 auf $20 \mathrm{mg} /$ Woche gesteigert wurde.

Der Hautzustand wurde anhand des Psoriasis Area and Severity Index (PASI) beurteilt. Bereits nach zwei Behandlungswochen war bei $38 \%$ der Patienten der Infliximab-Gruppe eine Verbesserung um mindestens $50 \%$ (PASI 50) sichtbar; $9 \%$ erzielten gar eine Ver- besserung um $75 \%$ (PASI 75). In der MTX-Gruppe erreichten dies lediglich 9 bzw. 0,5\% der Probanden. Noch deutlicher zeigte sich die Diskrepanz in den Wochen 16 und 26 mit 78 vs. $42 \%$ bzW. 77 vs. $31 \%$ Patienten mit PASI 75 . Einen Hautbefund entsprechend PASI 90 sah man bei 51 vs. $15 \%$ der Studienteilnehmer. Patienten, die bis zur Woche 16 nicht ausreichend auf die Therapie ansprachen (PASI < 50) wurden dem jeweils anderen Behandlungsarm zugeordnet. Unter den $29 \%$ Patienten aus der MTX-Gruppe, die mit Infliximab weiterbehandelt wurden, zeigten $73 \%$ nach 26 Wochen ein PASI-75-Ansprechen. Unerwünschte Therapieeffekte waren in beiden Behandlungsarmen vergleichbar; unerwartete Nebenwirkungen traten nicht auf. Auch die Lebensqualität verbesserte sich unter Infliximab signifikant, wie die Befragung mittels Dermatology Life Quality Index (DLOI) ergab.

dka

Nach Informationen von Essex Pharma 